\title{
FINITELY GENERATED SUBGROUPS AND THE CENTRE OF SOME FACTOR GROUPS OF FREE PRODUCTS
}

\author{
C.K. Gupta AND N. Romanovski
}

\section{Dedicated to Bernhard Neumann on his 90th birthday}

For groups of the type $F /[R, R]$, where $F$ is a free product, we prove a generalisation of a theorem of Karrass and Solitar on a finitely generated subgroup of a free product containing a nontrivial subnormal subgroup. We also describe the centre of the group $F /[R, R]$.

\section{INTRODUCTION}

We denote by

$$
F=\left(\underset{i \in I}{*} A_{i}\right) * X
$$

the free product of nontrivial groups $A_{i}(i \in I)$ and a free group $X$ with basis $\left\{x_{j} \mid\right.$ $j \in J\}$ such that $|I| \geqslant 1$ and $|J| \geqslant 1$. Define the rank of the decomposition (1) to be rank $F=|I|+|J|$. Let $R$ be a normal subgroup of $F$ such that $R \cap A_{i}=1(i \in I)$. Let $A=F / R ; G=F /[R, R]$, and $N=R /[R, R]$.

Karrass and Solitar [1] proved that if a finitely generated subgroup $H$ of the free product of two nontrivial groups contains a nontrivial subnormal subgroup of the free product then $H$ is of finite index. We prove a generalisation of the above result to groups of the type $F /[R, R]$ modulo the subgroup $R /[R, R]$.

THEOREM 1. Let rank $F \geqslant 2$. Let $H$ be a finitely generated subgroup of the group $G$ and let $C$ be a nontrivial subgroup of $H$ with a subnormal series:

$$
G=G_{1} \triangleright G_{2} \triangleright \ldots \triangleright G_{m} \triangleright C
$$

Then
(1) if $C \leq N$ then $|G: H N|<\infty$;
(2) if $C \leqslant N$ then $\left|G_{m} N:\left(H N \cap G_{m} N\right)\right|<\infty$.

Received 5th May, 1999

Copyright Clearance Centre, Inc. Serial-fee code: 0004-9727/99 \$A2.00+0.00. 
Our second result describes the centre of the group $F /[R, R]$. This generalises a result of Auslander and Lyndon [2] for the case $F=X$ which states that if $R$ is a nontrivial normal subgroup of a non-cyclic free group $F(=X)$ then the centre of $F /[R, R]$ is trivial if and only if $F / R$ is infinite.

THEOREM 2. Let $F$ be the free product (1) with rank $F \geqslant 2$ and $R \neq 1$. If either the group $A=F / R$ is infinite or, in the decomposition (1), the factor $X$ is not present, then the centre of the group $G$ is trivial. If, however, the group $A$ is finite and $X$ is nontrivial, then the centre of the group $G$ is a free Abelian group of rank equal to the rank of the free group $X$.

The proofs of these theorems essentially use a generalisation of Magnus and Shmel'kin embeddings for groups of the type $F /[R, R]$ which is defined and studied in [3].

\section{NOTATION AND PRELIMINARIES}

We use the following notation for a given group $G:[x, y]=x^{-1} y^{-1} x y, x^{y}=$ $y^{-1} x y, x^{\alpha_{1} y_{1}+\ldots+\alpha_{s} y_{s}}=\left(x^{\alpha_{1}}\right)^{y_{1}} \ldots\left(x^{\alpha_{s}}\right)^{y_{s}}$, for $x, y, y_{1}, \ldots, y_{s} \in G$ and $\alpha_{1}, \ldots, \alpha_{s} \in \mathbb{Z}$. We denote by $\langle U\rangle$ the subgroup generated by the set $U$ and define $[U, V]=\langle[u, v]| u \in U$, $v \in V\rangle$.

We assume for simplicity that the sets $I$ and $J$ of indices are finite, although all our proofs are valid without this assumption. Let $I=\{1, \ldots, n\}, J=\{n+1, \ldots, n+l\}$. Denote by $\bar{f}$ the canonical image in $A$ of an element $f \in F$. As canonical epimorphisms $F \rightarrow A, F \rightarrow G$ yield embeddings of subgroups $A_{i}(i \in I)$, we identify these subgroups with their images in $A$ and $G$ respectively. Denote by $\pi$ the canonical epimorphism $G \rightarrow A$.

Let $M$ denote the group of matrices $\left(\begin{array}{ll}A & 0 \\ T & 1\end{array}\right)$, where $T$ is a right $A$-module with a basis $\left\{t_{1}, \ldots, t_{n}, t_{n+1}, \ldots, t_{n+l}\right\}$. It is proved in [3] that the kernel of the homomorphism $\tau: F \rightarrow M$. defined by the mapping

$$
a_{i} \rightarrow\left(\begin{array}{cc}
a_{i} & 0 \\
t_{i}\left(a_{i}-1\right) & 1
\end{array}\right), x_{j} \rightarrow\left(\begin{array}{cc}
\bar{x}_{j} & 0 \\
t_{j} & 1
\end{array}\right) \quad\left(a_{i} \in A_{i}, i \in I, j \in J\right),
$$

is $[R, R]$. So we identify the groups $G=F /[R, R]$ and $F \tau$. We shall also need the following criterion from [3] for a matrix from $M$ to belong to the group $G$ :

$$
\begin{aligned}
& \left(\begin{array}{cc}
a & 0 \\
t_{1} u_{1}+\ldots+t_{n} u_{n}+\ldots+t_{n+l} u_{n+l} & 1
\end{array}\right) \in G \Leftrightarrow \\
& u_{1} \in\left(A_{1}-1\right) \cdot \mathbb{Z} A, \ldots, u_{n} \in\left(A_{n}-1\right) \cdot \mathbb{Z} A, \\
& u_{1}+\ldots+u_{n}+\left(\bar{x}_{n+1}-1\right) u_{n+1}+\ldots+\left(\bar{x}_{n+l}-1\right) u_{n+l}=a-1 .
\end{aligned}
$$


Lemma 1. Let $c \in G \backslash N, a=c \pi, 1 \neq t \in N$. Suppose that $a$ is an element of prime order $p$ and $t^{c-1}=1$. Then $t \in\left\langle c^{p}\right\rangle \cdot N^{1+c+\ldots+c^{p-1}}$.

Proof: As the elements of $N$ are represented in $M$ by unitriangular matrices and $N \triangleleft M$, we can identify $N$ with the corresponding submodule of the module $T$. We shall use the language of modules and instead of $t^{c-1}$ write $t(a-1)$. If we consider $T$ as an $\langle a\rangle$-module then it is a free module with a basis consisting of elements of the form, for instance, $t_{i} y$ where $i \in I \cup J$ and $y$ ranges over a fixed system of representatives of left cosets of the subgroup $\langle a\rangle$ in the group $A$. Hence, if $t(a-1)=0$ then the element $t$ is divisible by $1+a+\ldots+a^{p-1}$ in the module $T$, but in the general case a quotient does not belong to $N$.

Let $f \in F$ and $f \tau=c$. Consider the subgroup $L=\langle f, R\rangle$ of the group $F$. Note that $L / R$ is a subgroup of $A$ and $L /[R, R]$ is a subgroup of $G$. By the Kurosh subgroup theorem the group $L$ decomposes into a free product of some groups which are conjugates of $A_{i}$ and a free group. As $R$ has trivial intersection with any conjugate of a subgroup of $A_{i}$, we can replace the group $F$ by the group $L$ in our lemma.

So let $F=\langle f, R\rangle$. Then $A=F / R=\langle a\rangle$ is a cyclic group of prime order $p$. Consider an arbitrary element $h \in F \backslash R$ so that $\langle\bar{h}\rangle=A=\langle a\rangle$ and consequently, $(a-1) \cdot \mathbb{Z} A=(\bar{h}-1) \cdot \mathbb{Z} A$ and $\left(1+a+\ldots+a^{p-1}\right) \cdot \mathbb{Z} A=\left(1+\bar{h}+\ldots+(\bar{h})^{p-1}\right) \cdot \mathbb{Z} A$. Then $t(a-1)=0 \Leftrightarrow t(\bar{h}-1)=0$ and $N\left(1+a+\ldots+a^{p-1}\right)=N\left(1+\bar{h}+\ldots+(\bar{h})^{p-1}\right)$. Let $h=f^{s} r$, where $1 \leqslant s \leqslant p-1, r \in R$. We have $h^{p}=f^{s p} r\left(f^{s}\right)^{p-1}+\ldots+f^{n}+1$. It follows from this equation that

$$
\left\langle(h \tau)^{p}\right\rangle \leqslant\left\langle c^{p}\right\rangle+N\left(1+\bar{h}+\ldots+(\bar{h})^{p-1}\right)=\left\langle c^{p}\right\rangle+N\left(1+a+\ldots+a^{p-1}\right) .
$$

Similarly, $\left\langle c^{p}\right\rangle \leqslant\left\langle(\bar{h})^{p}\right\rangle+N\left(1+\bar{h}+\ldots+(\bar{h})^{p-1}\right)$, and hence

$$
\left\langle(\bar{h})^{p}\right\rangle+N\left(1+\bar{h}+\ldots+(\bar{h})^{p-1}\right)=\left\langle c^{p}\right\rangle+N\left(1+a+\ldots+a^{p-1}\right) .
$$

Therefore. if required, in our lemma we can replace the element $c=f \tau$ by any element $h \tau(h \in F \backslash R)$.

(a) We first consider the case when $F \neq X$, that is, the factors $A_{i}$ are present in the decomposition (1). Then every group $A_{i}(i \in I)$ must be cyclic of prime order $p$ and its canonical image in $A$ coincides with $A$. We may assume that $f=a \in A_{1}$. It is then possible to change the group $X$ by multiplying its basis elements by suitable powers of the element $a$ such that $X$ is contained in $R$. Let $t=t_{1} u_{1}+\ldots+t_{n+l} u_{n+l}$, where $u_{1}, \ldots, u_{n+l} \in \mathbb{Z} A$. As $t(a-1)=0$, every element $u_{i}(i=1, \ldots, n+l)$ is divisible by $1+a+\ldots+a^{p-1}$. Moreover, we know that

$$
u_{1} \in\left(A_{1}-1\right) \cdot \mathbb{Z} A=(A-1) \cdot \mathbb{Z} A, \ldots, u_{n} \in\left(A_{n}-1\right) \cdot \mathbb{Z} A=(A-1) \cdot \mathbb{Z} A .
$$

Therefore, the elements $u_{1}, \ldots, u_{n}$ are divisible by $a-1$. But then $u_{1}=0, \ldots, u_{n}=0$. Let

$$
u_{n+1}=v_{n+1}\left(1+a+\ldots+a^{p-1}\right), \ldots, u_{n+l}=v_{n+l}\left(1+a+\ldots+a^{p-1}\right) .
$$


The element $t^{\prime}=t_{n+1} v_{n+1}+\ldots+t_{n+l} v_{n+l}$ satisfies the criterion (3):

$$
\left(\bar{x}_{n+1}-1\right) v_{n+1}+\ldots+\left(\bar{x}_{n+l}-1\right) v_{n+l}=0 \cdot v_{n+1}+\ldots+0 \cdot v_{n+l}=0
$$

It follows that $t^{\prime} \in N$ and consequently, $t \in N\left(1+a+\ldots+a^{p-1}\right)$.

(b) Next, we consider the case when $F=X=\left\langle x_{1}, \ldots, x_{l}\right\rangle$ is a free group. By changing the basis of $F$ and the element $f$, if necessary, we may assume that $f=x_{1}$ and the remaining generators $x_{2}, \ldots, x_{l} \in R$. Then $R=\left\langle x_{1}^{p}, x_{2}, \ldots, x_{l}\right\rangle \cdot[F, F]$. Let $t=t_{1} u_{1}+\ldots+t_{l} u_{l}$. It follows from the condition $t(a-1)=0$ that

$$
u_{1}=k_{1}\left(1+a+\ldots+a^{p-1}\right), \ldots, u_{l}=k_{l}\left(1+a+\ldots+a^{p-1}\right)
$$

for some integers $k_{1}, \ldots, k_{l}$. Then we have

$$
\begin{aligned}
& t_{1} u_{1}=t_{1} k_{1}\left(1+a+\ldots+a^{p-1}\right)=\left(x_{1} \tau\right)^{p k_{1}}=c^{p k_{1}} \\
& \quad t_{2} u_{2}+\ldots+t_{l} u_{l}=\left(t_{2} k_{2}+\ldots+t_{l} k_{l}\right)\left(1+a+\ldots+a^{p-1}\right) \in N\left(1+a+\ldots+a^{p-1}\right) .
\end{aligned}
$$

We conclude that $t \in\left\langle c^{p}\right\rangle+N\left(1+a+\ldots+a^{p-1}\right)$. This completes the proof of Lemma 1. Proof of Theorem 1. Based on the theorem of Karrass and Solitar [1], we assume that $R \neq 1$. For a given element $t^{\prime} \in T$ denote by $\sigma\left(t^{\prime}\right)$ the support of $t^{\prime}$, that is, the set of all elements of $A$ on which $t^{\prime}$ depends. Let $B=H \pi$. Because the group $H$ is finitely generated, there is a finite system $\left\{y_{1} B, \ldots, y_{s} B\right\}$ of left cosets of the subgroup $B$ in the group $A$ such that for every matrix $\left(\begin{array}{cc}b & 0 \\ t^{\prime} & 1\end{array}\right) \in H$ the following inclusion holds:

$$
\sigma\left(t^{\prime}\right) \subseteq \Sigma=y_{1} B \cup \ldots \cup y_{s} B
$$

(1) Suppose, by way of contradiction, that the index $|G: H N|=|A: B|$ is infinite. Let $c \in C \backslash N$ and $a=c \pi$. We can assume that the element $a$ has either infinite order or its order is equal to a prime number $p$. As in Lemma 1 we identify $N$ with a corresponding submodule of the module $T$. Let $0 \neq t \in N, \sigma(t)=\left\{z_{1}, \ldots, z_{q}\right\}$. There is an element $y \in A$ such that $y \notin z_{j}^{-1} y_{i} B(j=1, \ldots, q ; i=1, \ldots, s)$. Then $\sigma(t y) \cap \Sigma=\emptyset$. Replacing $t$ by $t y$ we get $\sigma(t) \cap \Sigma=\emptyset$. It follows from the subnormality of the series (2) that $t(a-1)^{m} \in C$ so that $\sigma\left(t(a-1)^{m}\right) \cap \Sigma=\emptyset$. If $t(a-1)^{m} \neq 0$ we get a contradiction to inclusion (4).

So we may assume $t(a-1)^{m}=0$. Now $T$ is a free module over the group ring $\mathbb{Z}\langle a\rangle$. It then follows from the equation $t(a-1)^{m}=0$, that the element $a$ has finite order. We may assume that $a$ has order a prime number $p$ and that $t(a-1)=0$. Using the criterion (3), if the element $t$ is divisible in the module $T$ by some natural number then the quotient also belongs to $N$. So, we can assume that the element $t$ is not divisible by $p$. As $t(a-1)=0$, by Lemma 1 the element $t$ can be written in the form $t=\left(c^{p}\right) k+t^{\prime}\left(1+a+\ldots+a^{p-1}\right)$, 
where $k \in \mathbb{Z}, t^{\prime} \in N$. Let, for instance, $t=t_{1}(y u+\ldots)+\ldots$, where $y$ is a representative of a left coset of $\langle a\rangle$ in $A$ and the element $u \in \mathbb{Z}\langle a\rangle$ is not divisible by $p$. The coset $y B$ differs from $y_{1} B, \ldots, y_{s} B$ and $\sigma\left(c^{p}\right) \subseteq \Sigma$. Then $t^{\prime}=t_{1}(y v+\ldots)+\ldots$, where $v \in \mathbb{Z}\langle a\rangle, v\left(1+a+\ldots+a^{p-1}\right)=u$. Since $\left(1+a+\ldots+a^{p-1}\right)^{2}$ is divisible by $p$, the element $v$ is not divisible by $1+a+\ldots+a^{p-1}$. Hence, $v(a-1) \neq 0$, so that $v(a-1)^{m} \neq 0$ and $\sigma\left(t^{\prime}(a-1)^{m}\right) \nsubseteq \Sigma$. This is contrary to the condition $t^{\prime}(a-1)^{m} \in C \leqslant H$.

(2) If $C \leqslant N$ we can identify $C$ with the corresponding additive subgroup of the module $T$. Let $0 \neq t \in C$. Assume that the index

$$
\left|G_{m} N:\left(G_{m} N \cap H N\right)\right|=\left|G_{m} \pi:\left(G_{m} \pi \cap B\right)\right|
$$

is infinite. Then there is an element $a \in G_{m} \pi$ such that $\sigma(t a) \nsubseteq \Sigma$. Since $C$ is normal in $G_{m}$ it follows that $t(a-1) \in C$. On the other hand $\sigma(t(a-1)) \nsubseteq \Sigma$, contrary to (3). This completes the proof of Theorem 1.

PROOF OF THEOREM 2.

Lemma 2. Let $C(G)$ be the centre of $G$. Then $C(G) \leqslant N$.

Proof: Suppose $c \in G \backslash N$. We shall prove that the element $c$ does not commute with some element of $N$ and so $c \notin C(G)$. Let $a=c \pi$. If $|a|=\infty$ then for every nontrivial element $t \in N$ we have $t(a-1) \neq 0$, that is, the elements $t$ and $c$ do not commute. So, we can assume that the order of $c$ is finite and equal to a prime number $p$. Let $f \in F$ and $f \tau=c$. It follows from the conditions rank $F \geqslant 2, R>1$, and $R \cap A_{i}=1(i \in I)$, that $R$ is a free nonabelian group. Therefore, if we consider the Kurosh subgroup decomposition of the subgroup $\langle f, R\rangle$ of $F$ into a free product, then its rank is at least 2, and we can assume in our lemma that $F=\langle f, R\rangle$. Then $F / A=A=\langle a\rangle$ is a cyclic group of prime order $p$

(a) Let the groups $A_{i}$ be present in the decomposition (1), that is, $F \neq X$. In this case we can assume that $f=c=a \in A_{1}$ and $X \leqslant R$. Then each $A_{i}$ must be cyclic of order $p$ and its canonical image in $A$ must coincide with $A$. Let $n \geqslant 2$ and $A_{1}=\left\langle a_{1}\right\rangle, A_{2}=\left\langle a_{2}\right\rangle, \bar{a}_{1}=\bar{a}_{2}=a$. Consider the element $t=\left[a_{1} \tau, a_{2} \tau\right]=\left(t_{1}-t_{2}\right)(a-1)^{2}$. We have $t(a-1) \neq 0$. If $n=1$, that is, $F=A_{1} * X$, then rank $X \geqslant 1$. Let $t=x_{2} \tau=t_{2}$. We have again $t(a-1) \neq 0$.

(b) Let $F=\left\langle x_{1}, \ldots, x_{l}\right\rangle$ be a free group. Then it is possible to assume that $f=x_{1}$ and $x_{2}, \ldots, x_{l} \in R$. Let $t=x_{2} \tau=t_{2}$. Then $t(a-1) \neq 0$. Lemma 2 is proved.

Lemma 3. Let $A$ be an infinite group. Then $C(G)=1$.

ProOF: Based on the previous lemma, it is sufficient to prove that for every nontrivial element $t \in N$ there is an element $a \in A$ such that $t(a-1) \neq 0$. As $A$ is infinite we can choose an element $a$ such that $\sigma(t) \neq \sigma(t a)$. Then $t(a-1) \neq 0$. Lemma 3 is proved. 
Now we assume that the group $A$ is finite and let $d$ denote the sum of all elements of $A$.

Lemma 4. If $A$ is a finite group, then $C(G)=T d \cap N$.

PROOF: It is obvious that $T d$ is contained in the centre of the group $M$, therefore $C(G) \geqslant T d \cap N$. Assume that some element $t \in T$ does not belong to $T d$. Let, for example, $t=t_{1}\left(k_{1} b_{1}+k_{2} b_{2}+\ldots\right)+\ldots$, where $k_{1}, k_{2}, \ldots$ are integers, $b_{1}, b_{2}, \ldots$ are different elements of $A$ and $k_{1} \neq k_{2}$. If $a=b_{1}^{-1} b_{2}$, then $t a \neq t$. This means that the element $t$ does not centralise the group $G$. Hence, $C(G) \leqslant T d \cap N$. This proves Lemma 4.

The proof of Theorem 2 follows from Lemma 3 and the following lemma.

Lemma 5. Let $A$ be a finite group. Then the centre of the group $G$ coincides with an additive subgroup of the module $T$ generated by the elements $t_{n+1} d, \ldots, t_{n+l} d$.

ProOF: Let

$$
t=t_{1} u_{1}+\ldots+t_{n} u_{n}+t_{n+1} u_{n+1}+\ldots+t_{n+l} u_{n+l} \in C(G)=\operatorname{Td} \cap N
$$

As every element $u_{i}(i=1, \ldots, n+l)$ is divisible by $d$, it is possible to represent it in the form $u_{i}=k_{i} d, k_{i} \in \mathbb{Z}$. It follows from the criterion (3) that $u_{1}, \ldots, u_{n} \in(A-1) \cdot \mathbb{Z} A$. Then $u_{1}=\ldots=u_{n}=0$. So $C(G)$ is contained in the additive subgroup of the module $T$ generated by the elements $t_{n+1} d, \ldots, t_{n+l} d$. On the other hand, every element $t_{j} d(j \in J)$ which centralises $G$ and belongs to $G$ satisfies the criterion $(3):\left(\bar{x}_{j}-1\right) d=0$. This completes the proof of Lemma 5 and, in turn, the proof of Theorem 2.

\section{REFERENCES}

[1] A. Karrass and D. Solitar, 'On finitely generated subgroups of a free product', Math. $Z$. 108 (1969), 285-287.

[2] M. Auslander and R.C. Lyndon, 'Commutator subgroups of free groups', Amer. J. Math. 77 (1955), 929-931.

[3] N. Romanovski, 'On Shmel'kin embeddings for abstract and profinite groups', Algebra and Logic 38 (1999), 482-494.

Department of Mathematics

University of Manitoba

Winnipeg, Manitoba R3T 2N2

Canada

e-mail: cgupta@cc.umanitoba.ca
Institute of Mathematics

Novosibirsk 630090

Russia

e-mail: rmnvski@math.nsc.ru 